\title{
“IL NE LEUR MANQUE QUE LA PAROLE " ?
}

\author{
J.C. Quentel ${ }^{*}$ et J. M. Vidal ${ }^{* *}$
}

Qu'est-ce qui fonde la différence entre les comportements animaux et les conduites proprement humaines; cette différence est-elle simplement quantitative ou fondamentalement qualitative ? À cette double question, la philosophie, depuis qu'elle existe, n'a eu de cesse de proposer ses réponses. Aujourd'hui, diverses approches en sciences humaines, en éthologie et en psychologie comparative, s'emploient à lui trouver des solutions scientifiques. L'enjeu n'est pas mince puisqu'il s'agit de savoir si l'homme, qui relève incontestablement de l'animalité dans sa physiologie, participe ou non par ailleurs d'un autre ordre de réalité que les animaux dans ses modes de pensée et dans ses conduites en général. En d'autres termes, l'émergence de ce qu'on appelle le "culturel" correspond-elle à une discontinuité sans commune mesure avec celles repérables dans l'évolution des espèces, ou n'est-elle qu'un simple "surajout" dans la complexité de l'adaptation vitale ? (cf. Vidal, 1992).

Très généralement, les réponses apportées se focalisent sur le seul langage. En ce sens, le questionnement de nombre de chercheurs rejoint celui de Monsieur Tout le monde quand, devant son animal familier, il en vient à se dire qu'il ne lui manque somme toute que la parole. Le tout étant précisément de savoir ce que cette absence de parole signifie. Mais on se demandera de même, lorsqu'il y a vraisemblance de parole, ce que

* Professeur au Département des Sciences du Langage de l'Université de Rennes 2.

** Chercheur CNRS, URA 1031, Groupe de Recherches Sémiologiques et Thérapeutiques des Troubles de la Communication, Campus de Beaulieu, Bâtt 25, 35042 RENNES Cédex. 
celle-ci implique en termes de processus. Car au-delà de la simple constatation d'une apparence d'enchaînement de mots ou de signes gestuels, il s'agit de préciser le type de fonctionnement qui préside à leur production. Qu'en est-il, autrement dit, du langage dans ce qui le fonde véritablement comme phénomène spécifique? Plus encore, les autres registres de conduites - ceux qui concernent le maniement d'outils, les relations sociales ou les choix éthiques - sont-ils véritablement accessibles à l'animal ? À ce dernier, ne manque-t-il en fait que la parole?

Tout d'abord, parler n'est pas obligatoirement oraliser et encore moins écrire. Ceci n'a pas échappé à ceux qui ont su reconnaître les aptitudes des sourds-muets à parler en langage des signes, comme à ceux qui ont longtemps cherché l'équivalent d'un langage gestuel chez les animaux non humains, sans pouvoir le trouver (voir revue Lestel, 1995). On sait de plus que l'homme peut parler "intérieurement" sans traduire ce langage dans des expressions manifestes. Ainsi penser, c'est parler, et la clinique de l'aphasie, travaillée depuis plus d'un siècle, nous prouve qu'un trouble du langage entraîne irrémédiablement aussi un trouble de la pensée (Gagnepain, 1994).

Mais si parler n'est pas oraliser, alors l'animal qui ne parvient pas à articuler des sons est d'autant plus susceptible, dira-t-on, de disposer du langage au même titre que l'homme. D'où l'intérêt des procédures qui, depuis des décennies, tentent de dégager une utilisation du langage chez l'animal à partir de supports autres que le son. S'agit-il néanmoins d'un langage au même titre que chez l'homme? On notera déjà que la réponse à cette question varie selon la conception que le chercheur se fait du langage.

Aujourd'hui, les travaux des éthologistes confirment en tout cas amplement que les caractéristiques du langage, telles que les définit la linguistique moderne, ne s'expriment que chez l'homme. Dans les conditions naturelles, aucune espèce animale ne produit spontanément des sons ou des gestes qui équivaudraient à du langage. Nombre de chercheurs ont toutefois émis l'hypothèse que certains animaux disposaient en ce domaine de compétences bien supérieures à leurs performances habituelles - et que, s'ils ne parlaient pas, c'est qu'on n'avait pas su leur parler et leur apprendre à parler selon des modalités appropriées. Ils ont alors élaboré des dispositifs fort sophistiqués, dans leurs laboratoires, pour " donner la parole" à ceux qui leur paraissaient simplement en manquer. Ils ont certes pu, dans ces conditions, enrichir sensiblement le maniement des signaux et le répertoire d'expressions de leurs animaux; ils ne sont pas parvenus jusqu'ici à leur conférer véritablement le langage. 
$\mathrm{Au}$ mieux, les primates associent et juxtaposent plusieurs signaux, comme en témoigne ce chimpanzé qui, ayant appris à imiter divers gestes en associant l'un à la configuration d'un oiseau et un autre à celle de l'eau, a pu aussi les juxtaposer pour désigner un canard comme "oiseau-eau". Ils ne détachent cependant pas ces signaux des objets correspondants pour les articuler les uns aux autres et ne produisent jamais ni syntaxe (Terrace, 1985), ni morphologie (ensemble des morphèmes). Plus encore, les chercheurs qui tentent avec acharnement d'apprendre à parler à leurs chimpanzés, nous disent eux-mêmes que les plus doués d'entre eux gardent les plus grandes difficultés à associer plus de deux signaux ou à exprimer un simple “non" (Lestel, op. cit.). Précisons donc ce qu'il en est des caractéristiques essentielles du langage, telles que nous les révèle la linguistique moderne issue de Ferdinand de Saussure.

Pour produire des mots et des phrases, il ne suffit pas d'exprimer, ou a fortiori de répéter, une quelconque succession de sons ou de comportements étroitement liés à tel élément ou à telle situation de l'environnement. Même si le mainate imite nos mots - et que nous les reconnaissons comme tels -, il ne possède pas pour autant le langage, car il ne crée pas les mots. La faculté de langage suppose le dépassement d'une appréhension de cet environnement selon des liens immédiats entre indices et sens; elle implique l'accès à un univers médiat dans lequel un mot, quel qu'il soit, pourra désigner des choses qui n'ont entre elles aucun rapport naturel. Prenons par exemple en français le mot " bouton" : il n'existe aucun rapport immédiat entre le bouton de la fleur, celui de la porte, celui qui résulte de l'acné ou de la fièvre, celui de la chemise ou celui qui fait interrupteur. C'est le mot qui, ici, permet de regrouper tous ces sens sous le même terme ; le simple fait de changer de langue nous prouve par ailleurs qu'il n'y a dans ce regroupement rien de naturel.

En fait, tout élément de langage est foncièrement ambigu, impropre, même si, au moment où l'on désigne, on cherche à éliminer cette ambiguïté. En d'autres termes, le mot est foncièrement abstrait et ceci permet à l'homme de se donner le monde, c'est-àdire de se l'expliquer. Le sens découle donc du langage ; il présuppose une capacité mentale implicite que l'on appelle signe (Gagnepain, 1990 ; Jongen, 1993). Il s'agit d'une structure formelle qui organise implicitement notre dire et établit la relativité de l'élément de langage, quel qu'il soit. La définition d'un mot est en effet d'abord négative : elle est fonction de ses frontières avec les autres mots. Chaque élément n'est que ce que les autres ne sont pas, ainsi que l'a montré Saussure. De telle sorte qu'un mot, 
formellement, n'a pas de contenu en soi, mais une "valeur" qui se déduit par opposition ou par contraste de l'ensemble des autres mots.

La polysémie (ou multiplicité des sens d'un mot) et la synonymie (ou possibilité de reformuler ce que l'on a à dire), qui constituent les deux propriétés fondamentales du signe, attestent en permanence cette abstraction de l'élément de langage. Voilà ce dont l'animal n'est pas capable, quelles que soient les aptitudes cognitives hautement élaborées que nous révèlent les recherches menées sur les abeilles, pigeons et perroquets, dauphins et chimpanzés (Vauclair, 1992). Ceux-ci ne font jamais qu'associer immédiatement, certes d'une manière déjà complexe ; ils ne produisent pas l'élément de langage et ne sont pas capables de signe.

\section{SCHÉMA}

\section{LÉGENDE :}

Pour saisir la spécificité du langage, il faut le distinguer clairement d'un mode de connaissance immédiat du monde qui opère à partir d'une simple association univoque ou monosémique entre un indice (sonore visuel, odorant ou tactile) et un certain sens. Chez l'homme, les éléments de langage sont toujours polysémiques (porteurs de plusieurs sens) ; ils se définissent d'abord négativement, à partir des rapports qu'ils entretiennent entre eux. Le registre du culturel suppose donc un rapport médiat au monde.

À titre d'exemple, apparemment proche du système de communication par lequel un singe Vervet communique la présence d'un prédateur à ses congénères (voir encadré), prenons le cas d'un homme qui dit à son voisin: “ regarde l'aigle martial ”. Le sens de chacun des termes de ce message ne dépend formellement que des autres termes du système grammatical dans lequel ils s'inscrivent; autrement dit, leur signification ne résulte que de leur opposition et de leur contraste avec l'ensemble des autres éléments de langage dont ce locuteur dispose.

- "Regarde " n'a de sens que par opposition à écoute, sent, ..., comme à vois ou observe, ou encore à attention, fuis, cours...

- Il en va de même pour " aigle " qui n'a de sens que par rapport à cailloux, plantes et autres animaux ou nuages, mais aussi par contraste avec les autres unités " regarde" et " martial " du même message.

- Il faut de plus tenir compte de cette polysémie qui fait qu'un “ aigle ” peut désigner un oiseau rapace, aussi bien qu'un symbole plus ou moins stylisé, un humain mangeant goulûment, ou encore un personnage avide de pouvoir. Quant à " martial”, nous ne 
pouvons que laisser au lecteur le soin d'imaginer ce qu'une telle métaphore pourrait bien vouloir dire pour un Vervet...

\section{SCHÉMA}

\section{LÉGENDE :}

Les cris d'alarme des vervets n'apparaissent pas structuralement articulés les uns aux autres $(/ /)$, mais strictement liés ( ) aux configurations des objets ou prédateurs qu'ils désignent; leurs significations respectives, pour celui qui les émet comme pour ses congénères qui les reçoivent, découlent immédiatement de ce lien.

Au contraire, le langage humain implique cette articulation structurale à la fois phonologique (des sons en signifiants ou phonèmes) et sémiologique (des sens en signifiés, morphèmes et syntagmes), de telle manière que les signes qui résultent des rapports réciproques des signifiants et signifiés ( ) ne sont que secondairement reliés aux objets qu'ils désignent ( ); leurs significations résultent de leurs oppositions et contrastes $(<===>)$ avec l'ensemble des autres éléments de la structure langagière.

La parole qui manque alors à l'animal, c'est celle qui est permise par le signe. Seule la capacité de signe autorise une mise en rapports des éléments de langage les uns avec les autres, mise en rapports qui va en quelque sorte culminer dans le paradigme (catégorisation morphologique) et le syntagme (ordination de la phrase). De bonne heure, l'enfant en est capable, contrairement aux autres primates, même s'il fait ici des "fautes" qui traduisent, certes, sa mauvaise maîtrise de la langue de la société à laquelle il participe, mais qui confirment en même temps sa capacité à manier le langage, et jusqu'à ses conjugaisons et ses désinences, qu'il ne fait qu'adapter à sa manière (Quentel 1993).

Mais les animaux ne disposent pas non plus, par ailleurs, de ce qui fonde la technique proprement humaine, pas plus qu'ils ne vivent dans une société assimilable à celle de l'homme et qu'ils ne mettent en œuvre, enfin, une éthique.

Ainsi, si nombre d'animaux manipulent des instruments, pour pêcher des fourmis avec une brindille en l'effeuillant préalablement (Lawick-Goodall, 19..), ou pour casser des noix en les coinçant entre des pierres dont l'une peut paraître leur servir de "marteau" et l'autre d'“ enclume" (Boesch et Boesch-Achermann, 1991), ces instruments restent immédiatement liés à une seule tâche. Ces animaux n'utilisent pas cette brindille pour se curer les dents ou les oreilles, ou titiller quelque autre animal non comestible; ils ne s'amusent pas à lancer leur pierre tel un pavé dans la mare pour éclabousser les copains; ils ne se servent pas de leur " marteau" pour façonner quelque 
lance, pour fabriquer un autre marteau plus perfectionné et, encore moins, pour confectionner quelque ornement qui n'aurait plus pour fonction que sa valeur esthétique.

Jamais les animaux ne constituent de boîte à outil, produit d'une structure analogue au langage en ce qu'elle rassemble des éléments qui peuvent être utilisés pour plusieurs tâches ou qui permettent d'accomplir plus ou moins bien la même tâche. Le choix d'un quelconque élément dans une boîte à outil procède également par oppositions et par contrastes par rapport aux autres éléments disponibles et aux tâches qu'ils permettent d'accomplir. L'animal, quant à lui, ne fait qu'opérer des liens immédiats entre moyens et fins, sans pouvoir abstraire son instrument de la situation dans laquelle il s'inscrit au moment où il l'utilise. Il ne pourra lui conférer, à quelques variations immédiates près, une autre destination que celle que la conjoncture lui impose. Alors que pour l'homme, tout au contraire, l'usage d'un couteau, par exemple, ne se limitera aucunement à telle situation, ni à telle fonction. Il lui sera toujours possible, de plus, de s'en servir pour autre chose que pour couper ou de le garder pour le seul loisir de l'avoir dans sa poche.

De même, en ce qui concerne les relations sociales, certains animaux peuvent certes anticiper les réactions d'un congénère, mais ils ne peuvent pas tenir compte de ses préoccupations (cf. l'absence de theory of mind chez les chimpanzés, Premack et Woodruff, 1978). Par ailleurs, certains animaux peuvent s'associer avec des partenaires et même reconnaitre les liens de proximité entre un jeune et sa mère, ou entre des congénères qu'ils voient habituellement côte à côte (Cheney et Seyfarth, 1988 ; Dasser ,1988) ; ils peuvent encore reconnaître leur propre image dans un miroir (Gallup, 1977). Pourtant, ils n'accèdent pas à des rapports de parenté et à des relations d'alliance entre les membres de leur clan pas plus qu'avec ceux de clans plus ou moins éloignés ; ils ne situent pas leur propre place, dans leur filiation. Comment s'en étonner puisque ces rapports, relations et places relèvent encore d'une structure analogue à celle du langage (Lévi-Strauss, 1949) ? Si, par ailleurs, beaucoup d'animaux manifestent des "jeux" agressifs, sexuels ou de prédation, en signalant parfois à leur compagnon qu'ils jouent, ils ne font pas pour autant semblant et ne négocient aucunement avec leur partenaire - à travers une convention du type "on joue au papa et à la maman, au chat et à la souris, ... ”. L'homme, quant à lui, analyse son rapport à l'espèce et crée véritablement ses appartenances à la société tout autant que ses contributions. Il est au principe même de la Loi, laquelle est arbitraire, c'est-à-dire non nécessitée naturellement, et il n'a de cesse de négocier socialement des contrats. 
Enfin, quoiqu'on ait pu dire quant aux capacités éthiques des chiens (Lorenz, 1969) ou des chimpanzés (de Waal, 1992), ou à leur capacité de mentir (Byrne et Whitten, 1988), celles-ci paraissent ne relever que des fantasmes de chercheurs subjugués par une idéologie évolutionniste et continuiste qui voudrait que l'animal soit bumain et que l'homme ne soit qu'animal. Outre que de telles attributions relèvent d'un anthropomorphisme comme d'un zoomorphisme démesurés et suscitent de vives critiques parmi les éthologistes eux-mêmes (Kennedy, 1992), elles impliqueraient encore que les animaux dont il est question puissent effectuer des choix de valeurs en termes de beau et de laid, de bien et mal, de juste et injuste, de vrai et faux, de respect et de transgression, de bonne conscience ou de culpabilité... et qu'ils se conduisent effectivement en se donnant eux-mêmes de tels repères.

Technique, société et éthique supposent en fin de compte le même dépassement de l'immédiat que le langage et l'introduction par l'homme de rapports structuraux autres que ceux dont les animaux témoignent dans leur relation à leur environnement. Et contrairement à ce que l'on croit le plus souvent, il n'y a pas lieu de donner au langage quelque exclusivité ni quelque priorité au regard de ces diverses caractéristiques spécifiquement humaines. Aussi bien n’y a-t-il pas que la capacité de langage à distinguer l'homme de l'animal et à définir la culture. À ce dernier, il ne manquerait donc pas que la parole. L'homme médiatise à tous les niveaux son rapport au monde parce qu'il y introduit son analyse ; telle est l'essence même de cette Raison que les philosophes n'avaient fait que postuler et dont les sciences humaines, aujourd'hui, prouvent l'existence. Elles viennent notamment s'appuyer sur ce que les phénomènes cliniques révèlent par la négative, à travers l'aphasie, la psychose ou la névrose, par exemple.

Tout nous donne à penser que, après avoir confirmé l'opinion commune selon laquelle tel ou tel animal manquait au moins de parole, les recherches en éthologie et en psychologie comparative confirmeront aussi que les comportements des animaux se distinguent autant des automatismes d'une simple mécanique que des conduites proprement humaines. 


\section{Bibliographie}

BOESCH C. et BOESCH-ACHERMANN H., Les chimpanzés et l'outil, La Recherche, 1991, $233: 724-731$.

BYRNE W. et WHITEN A., Machiavellian Intelligence: social expertise and the evolution in monkeys, apes and humans, Oxford, Clarendon Press, 1988.

Cheney D.L. et SEYFARTH R.M., Assessment of meaning and the detection of unreliable signals by vervet monkeys, Anim. Behav., 1988, 36 : 477-486.

DE WAAL F.B.M., The Chimpanzee's sense of social regularity and its relation to the human sense of justice, American Behavioral Scientist, 1991, 34, 3 - 335-349.

Gagnepain J., Du Vouloir dire. Traité d'épistémologie des sciences humaines, tome 1, Du signe. De l'outil, Paris, Livre et Communication, 1990 (repris par De Boeck).

GagnePain J. (ss. la dir.), Pour une linguistique clinique, Rennes, P.U. Rennes, 1994.

GALLUP G.G., Self recognition in primates: a comparative approach to the bidirectional properties of conciousness, Amer. Psychol., 1977, 32 : 323-338.

JONGEN R., Quand dire, c'est dire. Initiation à une linguistique glossologique et à l'anthropologie clinique, Bruxelles, De Boeck, 1993, coll. Raisonnances.

KENNEDy J.S., The new anthropomorphism, Cambridge, Cambridge University Press, 1992. LAWICK-GOODALL 19..,

LESTEL D., Paroles de singes, Paris, La découverte, 1995.

LEVI-STRAuss C., Les structures élémentaires de la parenté, Paris, La Haye, Mouton, 1967 (1 éd. 1949).

LORENZ K., L'agression. Une histoire naturelle du mal, (Trad. de Das Sogenannte Böse, zur Naturgeschichte der Agression, par V. Fritsch), Paris, Flammarion, 1969.

PREMACK D. et WoOdrufF G., Does the chimpanzee have a theory of mind ?, Behav. Brain Sci., 1978, 4 : 515-526.

Quentel J.C., L'enfant. Problèmes de genèse et d'histoire, Bruxelles, De Boeck, 1993.

SAussure F. de, Cours de linguistique générale, Paris, Payot, 1982.

Terrace, H.S., In the beginning was the "Name", J. Amer. Psychol. Assoc., 1985, 40, 1011-1028.

VAUCLAIR, J., L'Intelligence de l'Animal, Paris, Seuil, 1992.

VIDAL, J.-M., Évolution des psychismes et évolution des organismes, in TORT P., Darwinisme et Société, Paris, P.U.F., 1992, pp. 519-548. 